\title{
Survey on Empirical Channel Models for WBAN
}

\author{
Sukhraj Kaur $^{1}$ and Dr. Jyoteesh Malhotra ${ }^{2}$ \\ ${ }^{1,2}$ ECE Department, GNDU RC-Jalandhar \\ ${ }^{1}$ sukhrajkaur91@gmail.com, ${ }^{2}$ Jyoteesh@gmail.com
}

\begin{abstract}
Wireless body area networks (WBAN) are expected to be a breakthrough technology in healthcare areas, telemedicine, and physical rehabilitation. The nature of the propagation of electromagnetic signals in complex shaped human body in the case of WBAN is expected to be very much different from that found in other environments, e.g. home, offices, streets, etc. So in order to design a high quality WBAN it is very important to examine and improve the propagation characteristics of channel models of WBAN. The aim of this paper is to provide an overview of different channel models of WBANs. The effect of channel behavior due to dynamics has been surveyed and challenges in channel modeling have been extracted.
\end{abstract}

Keywords: Wireless Body Area networks, Channel model, Path Loss

\section{Introduction}

A Wireless Body Area Network (BAN) is a combination of advanced nano and microtechnology components. It is a prominent technology which includes a wireless network usually formed from light-weighted small-sized ultra-low-powered intelligent sensors which are placed in (implantable) and around (wearable or placed close to) the body. These sensors measure, process, and transmit the body's physiological signals to a control unit $(\mathrm{CU})$ without constraining the activities of the wearer. Doctors can then access the collected data for real-time diagnosis and trigger treatment procedures in return. This gives patients greater mobility and increased comfort by freeing them from the need to be connected to hospital equipments that are required to monitor their conditions [1]. This also prevents patient from any kind of sudden attack.

So this new technology, WBAN holds much promise for future patient health monitoring. It's a novel information technology that is able to support early detection of abnormal conditions and prevention of its serious consequences. Many patients can benefit from continuous monitoring as a part of a diagnostic procedure, optimal maintenance of a chronic condition or during supervised recovery from an acute event or surgical procedure. BANs promise inexpensive, unobtrusive, and unsupervised ambulatory monitoring during normal daily activities for long periods of time.

However, in order for BANs to become ubiquitous and affordable, a number of challenging issues, such as integration, standardisation, system design, customisation, security and privacy, and social issues must be resolved.

In the literature, various campaigns have been carried out to compute the empirical channel models of WBAN. However there is a need to comprehend the characterization of these channels under varied channel conditions. So this paper is an attempt to bring together the work done in the area of WBAN channel model and their dynamics behaviour at one place. In doing so the open issues and challenges related to WBAN channel model development has been extracted as well.

In the next section, brief description of background of WBAN channel is followed by its literature survey. Subsequently, the present status of WBAN model and issues related to it has been given. The paper is finally concluded in the last section. 


\section{Background}

The development of the PAN (Personal Area Network) grew out of the work being done by a number of different groups working in MIT (Massachusetts Institute of Technology) in the 90's. The original groups wanted to interconnect information appliances that were carried on the body.

WBAN was first presented by T. G. Zimmerman in an article from 1996, but he gave these body networks the name wireless personal are network (WPAN) from the beginning. Later on PAN was redefined to be, e.g., cable replacement for up to 10 meters (e.g., Bluetooth) and the name WBAN evolved instead. IEEE 802 has established a Task Group called IEEE 802.15.6 in November, 2007 for the standardization of BAN. The purpose of the group is to establish a communication standard optimized for low power, high reliability application for BANs [19].

WBAN supports a wide range of medical and Consumer Electronics (CE) applications. In Healthcare, WBANs can not only detect anomalies, they can also improve the life style of hearing and visually impaired people. In Sport and fitness services, WBAN can help in improving fitness level of athletes. In Entertainment and gaming, Motion capturing techniques allow the tracking of the real-time information about motion of different body parts and use this information to direct the controller in videogames. New capabilities added by a WBAN will improve the performance of soldiers engaged in military operations. In the industrial framework WBANs can be used to monitor the surrounding environment in order to prevent the exposure to toxic gasses and radiations. In this last domain several applications could be envisioned, such as emotion detection, ambient intelligence, smart keys, identification and authentication. For instance, WBANs can be used to monitor people vital signs as a basis to develop a system that adapts the surrounding environment depending on the user condition and emotional status (music, lights, heating/cooling).

\subsection{Classification of WBAN}

WBAN system can be categorized into two parts from applications point of view.

- Medical BAN

- Non-medical BAN

Nonmedical BAN can be regarded as wearable consumer electronics and entertainment devices for the on-body communications. Medical BAN consists of implant devices and wearable medical systems to measure the health status of human body. Since the system requirement for varying applications are different. So medical BAN can be classified as:

- Wearable BAN

- Implantable BAN

Transmitter and Receiver are the integral part of the body area channel. The static path loss and impulse response models for the wearable and implantable wireless body area network include miniature antennas. These models are already contributed to IEEE 802.15.6 (task group on body area network). According to IEEE 802.15.6 document (Channel Model for Body Area Network); three types of nodes can be there [1]:

1. Implant node: A node that is placed inside the human body from immediately below the skin to further deeper inside the body tissue.

2. Body Surface node: A node that is placed on the surface of the human skin or at most 2 centimetres away.

3. External node (Gateway Node): A node that is not in contact with human skin (between a few centimetres and up to 5 meters away from the body). 


\subsection{Channel characterization}

To design and develop a competent and reliable system suitable for WBAN, a knowledge of a radio propagation channel as well as a simple and generic channel model are inevitably required. So in the following section various channel characteristics are discussed.

2.2.1. Model Types: In all cases, two types of model may be generated:

- A theoretical or mathematical model

- An empirical model

A theoretical model may be traceable back to the fundamental principles of electromagnetic propagation and will permit precise modeling of a specific situation at radio link level. It will require a detailed description of the propagation environment and is therefore probably not suitable for modeling of macro environments.

An empirical model may be traceable to an agreed set of propagation measurements and is intended to provide a convenient basis for statistical modeling of the channel. Compared to the theoretical model, the empirical model will use a greatly simplified description of the environment and, although statistically accurate at network level, will not be precise at link level.

Appropriate efforts could be made to ensure that the two sets of models are consistent with each other [1].

2.2.2. Fading: In the body area network communications, propagation paths can experience fading due to different reasons, such as energy absorption, reflection, diffraction, shadowing by body, and body posture. The other possible reason for fading is multipath due to the environment around the body. Fading can be categorized into two categories

- small scale fading

- large scale fading

Small scale fading is in concern with rapid fluctuations in the amplitude and phase of the received signal within a small local area in small period of time due to small changes in location of the on-body device or body positions [1].

Large scale fading refers to the signal attenuation due to mobility over larger areas. It occurs due to variation in distance covered by signal between antenna positioned on the body and external node because of diffraction from large surrounding objects.

2.2.3. Path Loss: In WBAN, path loss is both distance and frequency dependent .The path loss model between the transmitting and the receiving antennas can be represented as a function of the distance (d).Based on the Friis formula [3] in free space path loss in $\mathrm{dB}$ is expressed by equation (1) as follows:

$$
P L(d)=P L_{0}+10 \text { nlog }\left(\frac{d}{d_{o}}\right)
$$

Where $\mathrm{PL}_{\mathrm{o}}$ is the path loss at a reference distance $\mathrm{d}_{\mathrm{o}}$, and $\mathrm{n}$ is the path - loss exponent. The equation (1) represents increase in path loss with distance.

2.2.4. Shadowing: Shadowing is the process of blocking of signal by large objects which are encountered due to the variation in the environment surrounding the body or even due to movement of the body parts. It causes variation in path loss of signal from that of mean value for a given distance as shown in equation (1). When considering shadowing, the total path loss i.e. PL can be expressed by equation (2)[1]

$$
P L=P L_{0}+S
$$

Combining equation (1) and (2), total path loss is 


$$
P L=P L_{o}+10 \text { nlog }\left(\frac{d}{d_{0}}\right)+S
$$

2.2.5. Power Delay Profile: Because of multipath reflections, the channel response of a BAN channel looks likes a series of pulses. In practice the number of pulses that can be distinguished is very large, and depends on the time resolution of the measurement system. The power delay profile of the channel is an average power of the signal as a function of the delay with respect to the first arrival path.

\section{Literature Survey}

WBAN was first introduced in 1996 and since then the technology is continuously evolving. Several studies have been previously performed to investigate sending and receiving data through the human body. In the following, the most important studies reported in literature are introduced and their results are reviewed.

In 2003, two researchers from the University of Twente published a paper [5] which described the increasing demand of resources placed on the medical community, the rising costs of in-patient care, and the relative lack of out-patient monitoring. The paper defined "extra-BAN communication" (EBAN) as communication between a BAN and another network. The solution paper provided was MobiHealth, a BSN with EBAN connectivity to a $2.5 / 3 \mathrm{G}$ networks to provide out-patient monitoring of patients vital signs.

Jordi Agud Ruiz and Shigeru Shimamoto published a paper [9] in 2006 to investigate the transmission characteristics of the human body considering high radio frequency signals. The experiment results of the frequency domain response have shown that the received signal power decreases when the frequency increases, and the power decrease is faster when the distance between TX and RX is larger. From the results, it is believe that the frequency range up to $600 \mathrm{MHz}$ could be suitable for Intra body communication.

In 2007, Akram Alomainy et al. published a paper [13] in which they investigate the On-body propagation measurement using microstrip patch antennas at $2.45 \mathrm{GHz}$ in an anechoic chamber and in the lab. The measurements were taken for a range of body positions and postures, thereby allowing a subsequent investigation in the effects of body motion on the received signal. The results demonstrated that attenuation caused by trunk, head and jumper is around 19.2, 13.0, and $1.7 \mathrm{~dB}$, respectively. The dependency of system performance on the applied data rate was also demonstrated and it was shown that acceptable BER can be achieved for power levels as low as $-40 \mathrm{dBm}$ for low data rates commonly used in health monitoring applications and general wireless sensor networks.

Several investigations have been proposed in [2] by Attaphongse Taparugssanagorn et al. for UWB Channel Modeling for WBAN.In this study, it was found that for certain scenarios the UWB channel characteristics are distinctively varied depending on the gender or the medical condition of the subjects. The measurements obtained in this study are used to estimate channel parameters needed to build communication systems for WBAN medical applications.

Elisabeth Reusens et al. published a paper [10] in 2009 to characterize the path loss for different parts of the human body (arm, back, torso, and leg) obtained by measurements on actual humans. The path loss along the torso is the highest of the investigated body parts. They showed that single-hop communication is difficult in terms of energy consumption and multi-hop communication would be a better choice.

Simulative investigations have been carried out in [18] by Muhammad Shuja Uddin et $a l$. to study the path loss for static and dynamic nodes on different parts of the body. The movement of nodes with the body is accounted and the log normal propagation model for dynamic WBAN is proposed in this article. Increase in path loss of up to 15-20 dB for NLOS and around $5 \mathrm{~dB}$ for LOS situation has been observed with the dynamic nodes in 
WBAN. In addition different proposed energy models such as Cono et al, first order energy model, and its enhancements are discussed and analyzed in the context of WBAN. Finally, an energy model for the WBAN has been proposed. This model considers the values of power received and path loss from the previously proposed propagation model.

Matti Hämäläinen et al. in [17] discuss the UWB frequency band. The radio channel in a close proximity of a body has its own impact to the propagating signal. Due to the impact of human body on electromagnetic signal propagation, the energy of channel impulse responses less than 5-6 ns are decaying rapidly. On the other hand, the reflections from the environment will generate a large number of multipath components that are arriving later. The multipath propagated signal components coming from the environment can be separated from their WBAN counterparts due to their longer delays. The received signal power depends on sex, age, posture etc. of the person and also if the person has implants.

In 2012, paper [14] by Narendra Kumar, Alok Aggrawal and Nidhi Gupta deeply study the benefits of using wireless networks for medical applications. They have discussed about how these new Wireless technologies can be utilized in potential manner to get benefits for the human well being. Thus these technologies help us to design less intrusive Wireless sensor devices, which help us in ensuring human life.

Attaphongse Taparugssanagorn, Jani Saloranta, and Jari Iinatti published a paper [12] in IEEE conference which reviewed the research works on the channel characterizations and modelling for WBAN in particular for an indoor environment. The path loss is very high especially when the receive antenna is placed on the different side than the transmit antenna. The lognormal distribution turns to be the best fit for the small-scale fading rather than the traditional Rayleigh and Ricean distributions in the other environments.

Several investigations have been performed in [15] by Minseok Kim and Jun-Ichi Takada to characterized dynamic wireless on-body channels at three different frequencies in sub-GHz band. As is well known, multiple signals that arrive from different paths have different Doppler shifts and contribute to fast fading. However, this paper showed that the multipath contribution to fading was less significant in the light of the fact that the channel characteristics at different frequencies did not widely differ from one another. Therefore, it is concluded that the shadowing effect due to body movement is dominant over multipath fading due to the Doppler Effect in open office environments at sub-GHz bands.

In 2014, paper [16] by Haider A. Sabti \& David V. Thiel, the received signal level was recorded at 108 static body positions to simulate the human running and walking actions and for six different transmitter locations on the human body. The results showed that the sensor on the wrist provided the most reliable communications with another node attached to the chest.

\section{Present Status of WBAN Models}

In the WBAN, information propagates as electromagnetic waves from devices that are close to or inside the human body, but human body is never an ideal medium for the propagation of radio waves. The human body consists of complex shaped various types of tissues having different dielectric constant, permitivity, conductivity and characteristic impedance. Therefore, propagating wave experiences fading which is caused by energy absorption, reflection, diffraction and shadowing by body tissues and body posture. The multipath due to the environment around the body is also one of the factors causing fading which makes the channel models for WBAN different from the ones in the other environments. This model is a statistical model and uses all the measurements carried out by NICTA at $820 \mathrm{MHz}$. 
Table 1. NICTA specification and Allowed Range for WBAN

\begin{tabular}{|l|l|l|}
\hline Parameter & NICTA specification & Allowed Range \\
\hline Carrier Frequency $\left(\mathrm{f}_{\mathrm{c}}\right)$ & $820 \mathrm{MHz}$ & $420-2500 \mathrm{MHz}$ \\
\hline Sample Rate & $1 \mathrm{KHz}$ & $0.75-15 \mathrm{KHz}$ \\
\hline Velocity & $1.5-5.5 \mathrm{Km} / \mathrm{h}$ & $1.5-20 \mathrm{Km} / \mathrm{h}$ \\
\hline
\end{tabular}

A list of scenarios can be identified in which IEEE802.15.6 devices will be operating. These scenarios along with their description and frequency band are listed in Table 2 . The scenarios are determined based on the location of the communicating nodes (i.e. implant, body surface and external). The scenarios are grouped into classes that can be represented by the same Channel Models (CM).

Table 2. List of scenario and their description[1]

\begin{tabular}{|l|l|l|l|}
\hline SCENARIO & DESCRIPTION & FREQUENCY BAND & $\begin{array}{l}\text { CHANNEL } \\
\text { MODELS }\end{array}$ \\
\hline S1 & Implant to Implant & $402-405 \mathrm{MHz}$ & CM1 \\
\hline S2 & Implant to Body surface & $402-405 \mathrm{MHz}$ & CM2 \\
\hline S3 & Implant to external & $402-405 \mathrm{MHz}$ & CM2 \\
\hline S4 & $\begin{array}{l}\text { Body surface to Body } \\
\text { surface(LOS) }\end{array}$ & $\begin{array}{l}13.5,50,400,600,900 \mathrm{MHz} \\
2.4,3.1-10.6 \mathrm{GHz}\end{array}$ & CM3 \\
\hline S5 & $\begin{array}{l}\text { Body surface to Body } \\
\text { surface(NLOS) }\end{array}$ & $\begin{array}{l}13.5,50,400,600,900 \mathrm{MHz} \\
2.4,3.1-10.6 \mathrm{GHz}\end{array}$ & CM3 \\
\hline S6 & $\begin{array}{l}\text { Body surface to } \\
\text { external (LOS) }\end{array}$ & CM4 \\
& $\begin{array}{l}\text { Body surface to } \\
\text { external (NLOS) }\end{array}$ & $\begin{array}{l}900 \mathrm{MHz} \\
2.4,3.1-10.6 \mathrm{GHz}\end{array}$ & CM4 \\
\hline S7 & \multicolumn{2}{|l}{} \\
\hline
\end{tabular}

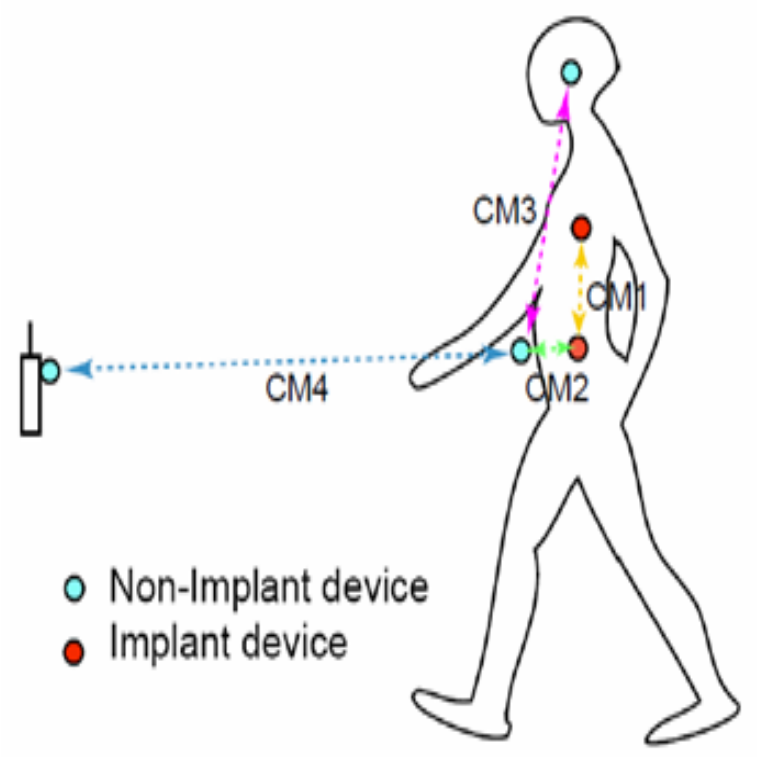

Figure 1. Possible communication links for BAN [1]

In wireless communications, it is well proved that the Rayleigh distribution is the best choice to model the envelope of the received signals when there are a large number of reflective and scattered paths with no LOS signal components. The Rice distribution is used to model the propagation environment where there are dominant non-fading 
components, such as a LOS propagation path. The Rayleigh distribution is a special case of Rice distribution and occurs when the Rican $\mathrm{K}$ factor is equal to zero. Nakagami-m distribution is known to be the best option for modeling the fading channel conditions that are severer than the Rayleigh distribution. The radio signals in WBANs may experience both types of small-scale and large-scale fading. However, in WBANs the small-scale fading is dominant compared to the large-scale fading due to the short distance between the transmitter and the receiver [3], [4]. In Table 3, best distributions are found corresponding to different body postures when sensors are placed at different body positions.

Table 3. The Best Fitting Distribution for Dynamic Channel at $4.5 \mathrm{GHz}$

\begin{tabular}{|l|l|l|l|}
\hline POSITIONS & STILL & WALKING & UP/DOWN \\
\hline Right Wrist & Normal & Weibull & Weibull \\
\hline Right upper arm & Log-normal & Weibull & Weibull \\
\hline Right ear & Normal & Log-normal & Log-normal \\
\hline Head & Weibull & Log-normal & Weibull \\
\hline Ear & Log-normal & Weibull & Weibull \\
\hline Shoulder & Log-normal & Log-normal & Weibull \\
\hline Right Rib & Log-normal & Log-normal & Weibull \\
\hline Left Waist & Normal & Log-normal & Weibull \\
\hline Right Thigh & Log-normal & Log-normal & Weibull \\
\hline Right Ankle & Log-normal & Weibull & Weibull \\
\hline
\end{tabular}

From Table 3, it is concluded that

- Normal distribution seems to fit the still posture best, but it can be seen that fittings with any distributions have large from the PDFs.

- Log-normal distribution shows good match in cases of still postures and small movements such as walking posture in case of head, right ear, chest, right rib, left waist, right thigh, and stand up/down posture in case of head.

- Weibull distribution can represent much better large movement behaviors such as walking posture in case of right wrist, right upper arm, shoulder, right ankle, and all stand up/down postures except for head[20].

In On-body wireless body area network, signal transmitted by sensors placed on different body positions are deteriorated due to shadowing by the human-body. This is seen by path losses for set of activities (standing, walking and running) given in Table 4 and 5 for different body positions at $2.36 \mathrm{GHz}[8]$.

Table 4. Average Path Loss for Receiver at Right Hip

\begin{tabular}{|l|l|l|l|}
\hline \multirow{2}{*}{ Transmitter at: } & \multicolumn{3}{|c|}{ ACTIONS } \\
\cline { 2 - 4 } & Standing & Walking & Running \\
\hline Chest & high & medium & Medium \\
\hline Right Wrist & low & low & Very low \\
\hline Left Wrist & Very high & medium & Medium \\
\hline Right Ankle & high & medium & Medium \\
\hline Left Ankle & Very high & medium & Medium \\
\hline Back & Very high & $\begin{array}{l}\text { Very } \\
\text { high }\end{array}$ & High \\
\hline
\end{tabular}


Table 5. Average Path Loss for Receiver at Chest

\begin{tabular}{|l|l|l|l|}
\hline \multirow{2}{*}{ Transmitter at: } & \multicolumn{3}{|c|}{ ACTIONS } \\
\cline { 2 - 4 } & Standing & Walking & Running \\
\hline Back & Very high & Very high & Very high \\
\hline Right Wrist & Very high & High & medium \\
\hline Right Ankle & high & High & high \\
\hline
\end{tabular}

The results showed that the sensor on the wrist provided the most reliable communications.

So from the above discussion, the detailed information about the WBAN models is obtained which will act as ready reference for the researchers in their future work in the very same field.

\section{Open Issues}

Wireless medical sensors should satisfy the main requirements such as wear ability, reliability, security, interoperability, functionality and some corresponding challenges like time synchronization, energy efficiency (low power constraint),interference, physical layer communication, bandwidth constraint, regulatory issues and low cost. However the major issues of WBAN channel which need immediate attention of researchers are as follow:

Wear ability: To achieve non-invasive continuous health monitoring, wireless medical sensors should be lightweight and small. The size and weight of sensors is predominantly determined by the size and weight of batteries. In turn a battery's capacity is directly proportional to its size which leads to the challenges of energy efficiency.

Reliable communication: In WBANs reliability is of utmost importance for medical applications as these are subject to very sensitive information about patients. It could be dangerous, even fatal, for false readings to appear on a patient's glucose monitor output.

Security: The problem of security arises at all three tiers of a WBAN-based telemedical system. At the lowest level, wireless medical sensors must meet confidentiality requirements mandated by the law for all medical devices and must guarantee data integrity. The strict security mechanisms are desirable that would prevent malicious interaction with the systems.

Interference: It is not unreasonable to expect hundreds of thousands of these networks in place in the next decade. This can lead to the problem of interference between wireless networks in people standing next to each other or even the possibility of colliding signals within one person.

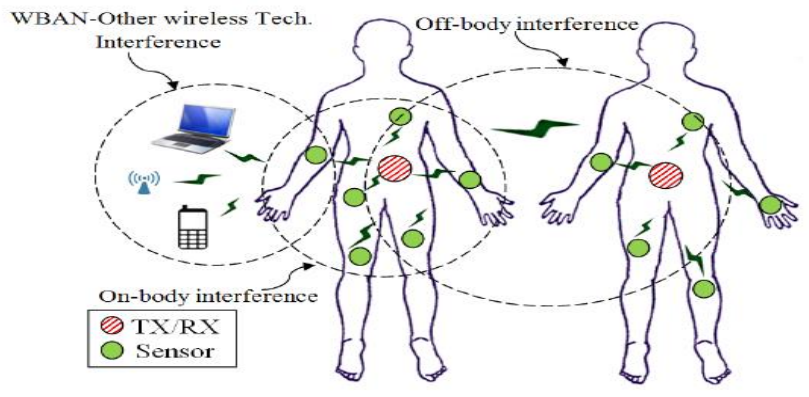

Figure 2. Interference in WBAN[11] 
Fading: In the WBAN, fading is caused by environment inside or outside the body. It occurs due to variation in the relative position between transmitter and receiver and because of variation in distance covered by signal between antenna positioned on the body and external node (office or hospital) because of diffraction from large surrounding objects.

Energy Efficiency: Energy consumption is one of the fundamental design constraints in wireless sensor networks since sensor nodes have size restrictions and can be operated by either battery or wireless power transfer. To extend each node's lifetime, it is necessary to reduce power dissipation as much as possible. If the node is implanted in the body, it is not practical to replace the battery as often as would be required.

Bandwidth: The frequency range selected for communications plays an important role in the design and performance of WBAN as there is a direct relationship between frequency and tissue warming. The higher the frequency of the EM signal, the higher is its absorption by the tissue and more the tissue warming. Hence it is desirable to use lower frequencies for communications. However, the lower the frequency, the larger will the antenna dimensions have to be. Therefore there is a trade-off between antenna dimensions and greater tissue warming. To avoid excessive radio interference as well as having to obtain FCC licenses, it may be advisable to use the designated, unlicensed ISM frequency bands. [7]An FCC regulation, however, makes it mandatory for all communication on the ISM frequency band to use spread spectrum techniques. This further increases the complexity of the transceivers on the biosensors.

The selection of frequency band is one of most important issues of WBAN. Generally available frequencies are regulated by communication authorities in different countries. Also frequency regulations for medical applications were issued in many countries or regions. Table 6 summarizes the available frequency bands for wireless medical telemetry system (WMTS), industry science and military(ISM) system, ultra wideband (UWB) system and medical implant communication system (MICS) in EU, Japan, and USA. The measured physiological parameters and other patient related information are transmitted via RF communication between a patient-worn transmitter and a remote monitoring unit.

Table 6. Frequency Regulation for WMTS and MICS

\begin{tabular}{|l|l|l|}
\hline FREQUENCY & $\begin{array}{l}\text { FREQUENCY } \\
\text { BAND }\end{array}$ & $\begin{array}{l}\text { REGULATING } \\
\text { COUNTRIES }\end{array}$ \\
\hline MICS & $402-405$ & $\begin{array}{l}\text { EU, Korea, } \\
\text { Japan, USA }\end{array}$ \\
\hline \multirow{2}{*}{ WMTS } & $420-429,440-449$ & Japan \\
\cline { 2 - 3 } & $\begin{array}{l}608-614,1395- \\
1400,1427-1429.5\end{array}$ & USA \\
\hline ISM & 2400 & World Wide \\
\hline UWB & $3100-10600$ & $\begin{array}{l}\text { EU, Japan, } \\
\text { Korea, USA }\end{array}$ \\
\hline
\end{tabular}

Low Cost: [8] Today's wireless sensor systems cost around 100€ or higher. A major reason for this is the low volume of the market so far, but another more technical reason is that there are no commercially available packaging technologies that can efficiently integrate such heterogeneous components as batteries, MEMS, processors, and radios in a single package.

Functionality: Most of today's bio sensors act as simple gateways, passing on the information to a central hub where the data is converted into actionable information. In addition, [6] intelligence is required for the system to make decisions depending on the 
status of the environment, thus enabling context awareness. Finally, embedded intelligence opens the door to closed-loop systems, providing action or feedback to the user.

\section{Conclusion}

WBAN provides a wide range of application to improve the quality of life. It is continuously evolving and is under active research. Several investigations had already been carried out in this field and lots of challenges are still need attention from the research community especially in channel modeling of WBAN. This work provides a comprehensive survey of current state-of-the-art in WBAN channel modeling and its behavioral dependence on channel dynamics. Major issues related to channel modeling has been extracted and a possible future direction of research in the development of WBAN highlighted. It is expected that this work will act as ready reference for researchers and academicians working for further development of WBAN based system.

\section{Acknowledgements}

We express our profound gratitude and deep regard to our guide for his exemplary guidance, monitoring and constant encouragement throughout the course of this project. We owe our debt of gratitude to our department for the vision and foresight which inspired us to conceive this work. Lastly, we thank almighty, our parents and teachers for their encouragement without which this project would not be possible.

\section{References}

[1] K. Y. Yazdandoost and K. Sayrafian-Pour,"Channel Model for Body Area Network(BAN)", IEEE802.15.6 technical contribution, document ID: 15-08-0780-09-0006, 27 April, 2009, pp. 41-56.

[2] A. Taparugssanagorn, C. Pomalaza-Ráez, A. Isola, R. Tesi, A.Rabbachin, M. Hämäläinen, J. Iinatti, "UWB Channel Modelling for Wireless Body Area Networks in Medical Applications," the $3^{\text {rd }}$ International Symposium on Medical Information and Communication Technology, Montreal, Canada, 2009.

[3] H. Bai and M. Atiquzzaman, "Error modelling schemes for fading channels in wireless communication: A survey" IEEE Communications Surveys and Tutorials, vol. 5, pp. 2-9, Dec. 2003.

[4] M. G. Shayesteh, "Exact symbol and bit error probabilities of linearly modulated signals with maximum ratio combining diversity infrequency nonselective rician and rayleigh fading channels," IET Communications, vol. 5, pp. 12-26, Jan. 2011.

[5] Konstantas D, Herzog R. Continuous monitoring of vital constants for mobile users: The MobiHealth approach. 25th Annual International Conference of the IEEE EMBS. Piscataway, NJ: IEEE, 2003; $3728-3731$.

[6] Schwiebert L, Gupta Sandeep K. S. , Weinmann Jennifer, "Research Challenges in Wireless Networks of Biomedical Sensors", Proceedings of the 7th annual international conference on Mobile computing and networking, ACM New York, NY, USA (C2001

[7] V. KAUR, J. MALHOTRA: Performance Evaluation ofM-ary Modulations through WBAN Channel IMACST: VOLUME 2

[8] D. Smith, D. Miniutti, T. A. Lamahewa, and L. Hanlen, "Propagation models for body area networks: A survey and new outlook," to appear in IEEE Antennas and Propagation Mag., Dec. 2013.

[9] J. Ruiz and S. Shimamoto, "Experimental evaluation of body channel response and digital modulation schemes for intra-body communications," in IEEE International Conference on Communications 2006. vol. 1, June 2006, pp. 349-354.

[10] E. Reusens, W. Joseph, G. Vermeeren, L. Martens, B. Braem, C. Blondiam B. Latr'e and I. Moerman, "Characterization of on-body communication channel and energy efficient topology design for wireless body area networks," IEEE transaction on information technology in biomedicine, Vol. 13, No. 6, Nov 2009

[11] Samanesh Movassaghi et Al."Wireless Body Area Networks: A Survey"IEEE communication survey and tutorials 2013

[12] A. Taparugssanagorn, A. Rabbachin, M. Hämäläinen, J. Saloranta, J. Iinatti, ”A Review of Channel Modelling for Wireless Body Area Network in Wireless Medical Communications," in Proc. Wireless Personal Multimedia Communication Symposium, Saariselkä, Finland, 2008. 
[13] Akram Alomainy et al. "Statistical Analysis and Performance Evaluation for On-Body Radio Propagation With Microstrip Patch Antennas" IEEE TRANSACTIONS ON ANTENNAS AND PROPAGATION, VOL. 55, NO. 1, JANUARY 2007,pp 245-248

[14] Narendra Kumar, Alok Aggrawal and Nidhi Gupta "Wearable Sensors for Remote Healthcare Monitoring System", International Journal of Engineering Trends and Technology- Volume3 Issue12012.

[15] Minseok Kim and Jun-Ichi Takada "Characterization of Wireless On-Body Channel Under Specific Action Scenarios at Sub-GHz Bands"IEEE TRANSACTIONS ON ANTENNAS AND PROPAGATION, VOL. 60, NO. 11, NOVEMBER 2012.

[16] Haider A. Sabti \& David V. Thiel , Procedia "A study of wireless communication links on a body centric network during running Engineering 72 ( 2014 ) 3 - 8.

[17] Matti Hämäläinen et al. "On the WBAN Radio Channel Modelling for Medical Applications”, EuCAP 2011, pp 3120-3124.

[18] Muhammad Shuja Uddin et al. "Wave Propagation and Energy Model for Dynamic Wireless Body Area Networks".

[19] K.S. Kwak, S. Ullah and N. Ullah, "An Overview of IEEE 802.15.6 Standard," 3rd International Symposium on Applied Sciences in Biomedical and Communication Technologies, Rome, 7-10 November 2010, pp. 1-6.

[20] Minseok Kim et al. , "Statistical property of dynamic BAN channel gain at 4.5 GHz," IEEE 802-.15-080489-01-0006, September 2008.

\section{Authors}

Er. Sukhraj Kaur received B.Tech. degree in Electronics and Communication from Guru Nanak Dev University, Regional Campus, Jalandhar in 2014 and is pursuing M.Tech. in ECE (Specialization in Communication Systems) from GNDU, Regional Campus, Jalandhar. Her research area includes Statistical Modeling of Fading Channels in WBAN. She has published 3 research papers in International Journals so far.

Dr. Jyoteesh Malhotra B.Eng., M.Tech., PhD. is involved in teaching and research at Electronics and Communication Department, Guru Nanak Dev University, Regional Campus, Jalandhar. His research areas of interest include Statistical modeling of Fading Channels, Fading mitigation techniques in Wireless Communication and Optimization of WBAN air interface. Dr. Malhotra has more than 90 research publications and authored 02 books. He is a life member of I.S.T.E. and editorial board member of many International Journals of repute. 
International Journal of Future Generation Communication and Networking Vol. 8, No. 2 (2015) 